\title{
Can Pharmacological Inhibitors and Antibodies Against m CRP Suppress Interactions with Platelets and Leukocytes-Dampening Inflammation after Stroke
}

\author{
RS Baradie ${ }^{1}$, AM Algohary $23 *$, Esam S Al-Malki ${ }^{4}$, Yasser HS Babair ${ }^{5}$, Abdulrahman M Alsulaiman ${ }^{6}$, \\ Suliman A Alsagaby ${ }^{7}$ and Slevin $\mathbf{M}^{8}$ \\ ${ }^{1}$ Department of Medical Laboratory Sciences, College of Applied Medical Sciences, Saudi Arabia \\ ${ }^{2}$ Chemistry Department, Sciences college zulfi, Saudi Arabia
}

${ }^{3}$ Pharmaceutical Chemistry Department, National Organization for Drug Control and Research (NODCAR), Egypt

${ }^{4}$ Medical Laboratory Department, College of Applied Medical Sciences, Saudi Arabia

${ }^{5}$ Director of Northern Armed Forces Hospital's Ministry of Defense Medical Services, Saudi Arabia

${ }^{6}$ Consultant in Molecular \& Medical Virology. PSMMC Riyadh, Saudi Arabia

${ }^{7}$ Assistant professor, Department Medical Laboratory Sciences, College of Applied Medical Sciences, Saudi Arabia

${ }^{8}$ Cell Pathology School of Life Sciences Manchester Metropolitan University Manchester, UK

*Corresponding author: AM Algohary, Chemistry Department, Sciences college zulfi, Majmaah University, Arabia, Pharmaceutical

Chemistry Department, National Organization for Drug Control and Research (NODCAR), Giza, Egypt

\section{ARTICLE INFO}

Received: 幽 May 01, 2019

Published: May 08, 2019

Citation: RS Baradie, AM Algohary, Esam S Al-Malki, Yasser HS Babair. RS Baradie, AM Algohary, Esam S Al-Malki, Yasser HS Babair. Can Pharmacological Inhibitors and Antibodies Against $m$ CRP Suppress Interactions with Platelets and Leukocytes-Dampening Inflammation after Stroke. Biomed J Sci \& Tech Res 17(5)-2019. BJSTR. MS.ID.003062.

Keywords: Monomeric C-reactive protein m CRP; Alzheimer's Disease; Neuroinflammation; Antibody

\section{ABSTRACT}

In the last decade we and others have previously showed the importance of $m$ CRP in the induction of inflammation and in addition, the prevalence of the monomeric C-reactive protein ( $\mathrm{m} \mathrm{CRP}$ ) form in the brains of individuals with dementia, after ischaemic stroke and following other brain vascular damage or degeneration. Since neuroinflammation may be a key trigger/cause of later dementia, here we carried out a simple IHC study on a small cohort of brain samples, using a monoclonal (8C10 clone) highly specific anti-m CRP antibody to investigate its expression in vascular regions of damage highlighting the associated tissue morphology. We showed that $\mathrm{m}$ CRP was present in specific brain regions with medium sized cerebral vessels showing high intraluminal content that was associated with leakage in to the local tissue. Where this occurred, cells with the morphology of macrophages and glia, as well as smaller micro vessels became m CRP-positive showing evidence of a perpetuation of inflammation. In conclusion, vascular injury or leakage from any cause appears to be associated with $\mathrm{m} \mathrm{CRP} \mathrm{accumulation} \mathrm{and} \mathrm{leakage} \mathrm{into} \mathrm{brain} \mathrm{tis-}$ sue and this could be a critical element associated with enhanced neuroinflammation and later neurodegeneration and dementia.

\section{Introduction}

Alzheimer's disease (AD) is the greatest public formula of dementia disorder [1]. The modern therapeutic means existing to physicians serve mainly to improve patient symptoms and are incapable to stop the progressive decline in cognitive function that is the mark of $\mathrm{AD}$ [1]. It is hoped that new generations of agents will be able to slow or even halt the progression of dementia. However, it is likely that any significant therapeutic advance will rely upon an improvement in our current understanding of the pathophysiology of $\mathrm{AD}$, enabling novel treatment approaches to be developed. We have formerly revealed that (m CRP) was deposited in the brain extracellular matrix (ECM) of patients after critical stroke [3]. Furthermore, Cerebrovascular disease, neurovascular dysfunction 
and cerebral blood flow abnormalities are now recognized as critical influences in the pathophysiological development of AD, damaged, blocked or in patent vessels having a severe effect on the function of local neurovascular units (with approximately $80 \%$ of $\mathrm{AD}$ brains at post-mortem shown to have significant vascular abnormalities). Damage to the deep penetrating vessels within the brain such as following lacunar stroke, ischaemic stroke or head trauma, may lower the threshold for development of vascular and other (mixed) dementia, particularly in younger patients $[4,5]$. Also, in the last investigation we determine localization within the brain and indicators of previous stroke or vascular disruption in nonhuman samples [6]. Chronic neuroinflammation is strongly associated with later development of cognitive decline and dementia/Alzheimer's disease Selles MC et al. [7] and in addition, acute events such as ischaemic stroke, traumatic brain injury, and intracerebral haemorrhage as well as smaller vascular insults associated with for example lacunar stroke predispose individuals significantly to neurodegenerative complications Rensma et al. [8], Vinters et al. [9].

C-reactive protein has long been known to represent a useful marker of both acute and chronic inflammation Slevin et al. [6] however only in recent years has it, and particularly its monomeric form (m CRP-monomeric C-reactive protein) been shown to be biologically active within the brain and strongly associated with neuroinflammation and degeneration if not directly a causative factor in development of dementia Luan and Yao [10]. The involvement of $\mathrm{m}$ CRP in angiogenesis and vascular abnormalities Boras E et al. [11] prompted us here to examine small cohort of human brain samples from individuals after death who had clinical and pathological evidence of the existence of stroke and/or dementia. We analyzed specifically the localization of $m$ CRP within vascular regions of the brain. Prior studies have attempted to shown that $\mathrm{m} \mathrm{CRP}$ is deposited in significant quantities within the brain parenchyma after stroke, and we have recently identified a possible role of this protein in supporting neurodegeneration and aberrant vascular development. Recent clinical trials for AD have focused on attempts to remove pathological substrates e.g. amyloid-p, tau and neurofibrillary tangles (NFTs). So far, they have been unsuccessful. Here we can fix two hypothesize First one is vascular disruption and concomitant releasing of $\mathrm{m}$ CRP within the human brain tissue could increase on-going neurological damage via stimulation of neuroinflammation and from direct consequences of its action on both neuronal and vascular cells. Second, there are no pharmacological agents with regulative approval for use in the treatment of vascular dementia and novel approaches are desperately needed.

\section{Methods}

Alzheimer's tissue (post mortem) from 10 patients and was obtained from the Brain Bank in Bristol (UK). Tissue sections were obtained from blocks of the cerebral cortex, specifically, from the frontal lobe, parietal lobe and occipital lobe. Details are shown in Table 1 . The AD cases all had a history of progressive dementia with or without Stroke and were selected on the basis of a diagnosis according to CERAD of 'definite $\mathrm{AD}$ and a Braak tangle stage of V-VI; according to NIA-Alzheimer's Association guidelines, $\mathrm{AD}$ neuropathological change was considered a sufficient explanation for the dementia in all cases. The normal controls had no history of dementia, few or no neurotic plaques, and no other neuropathological abnormalities Mirra et al; Braak et al; Mortine et al. [12-14]. Samples were dissected into infarcted (identified with 2, 3, 5-triphenyltetrazolium chloride), peri-infarcted and normal looking unaffected tissue as previously described. Periinfarcted tissue showed structural integrity but was characterized by oedema, altered morphology of the neurons (some showing changes of apoptosis), inflammatory macrophage infiltration and angiogenesis. Tissue from the contralateral hemisphere served as a control. Samples were dissected into $2 \mathrm{~mm}$ diameter pieces and either frozen in liquid nitrogen at $-70^{\circ}$ or fixed in $10 \%$ buffered saline prior to paraffin embedding.

Table 1: patients / samples F=frontal; $\mathrm{BG}=$ basal ganglia; $\mathrm{OCC}=$ occipital; $\mathrm{PAR}=$ parietal; $\mathrm{AD}=\mathrm{Alzheimer}$ 's disease; $\mathrm{CVD}=$ cardiovascular disease; $\mathrm{VaD}=$ vascular dementia; $\mathrm{CAA}=$ cerebral amyloid angiopathy; $\mathrm{TBI}=$ traumatic brain injury. Results: We showed that monomeric-C-reactive protein deposition was highest in those regions affected by stroke or vascular disruption, and that within those same areas, there was more interaction and co-localization between major classical proteins of neurodegeneration $(\beta$-amyloid and tau).

\begin{tabular}{|c|c|c|c|c|}
\hline Number & Age & Sex & Diagnosis-1 & Diagnosis-2 \\
\hline $893 \mathrm{~F}$ & 71 & $\mathrm{M}$ & $\mathrm{AD}$ & CAA \\
\hline 883BG & 91 & $\mathrm{~F}$ & CVD & - \\
\hline 9160CC & 72 & $\mathrm{~F}$ & Va D & MELANOMA \\
\hline $850 \mathrm{~F}$ & 79 & M & $\mathrm{AD}$ & CVD \\
\hline 798PAR & 95 & $\mathrm{~F}$ & CVD & $\mathrm{AD}$ \\
\hline 9540CC & 84 & $\mathrm{~F}$ & CVD & - \\
\hline $893 \mathrm{~F}$ & 71 & $\mathrm{M}$ & $\mathrm{AD}$ & CAA \\
\hline 963PAR & 71 & M & $\mathrm{AD}$ & CAA \\
\hline 855BG & 93 & $\mathrm{~F}$ & TBI & CVD \\
\hline $815 \mathrm{~F}$ & 70 & M & $\mathrm{AD}$ & - \\
\hline
\end{tabular}




\section{Results}

Of the 10 patient samples examined, 7 showed significant macroscopically visible and specifically $\mathrm{m}$ CRP positive regions that were associated with vascular structures and abnormal looking brain tissue parenchymal morphology. The major highlights were as follows:

A. 893-F: there is notable m CRP staining around blood vessels and evidence of positively stained macrophages and microglia in small regions of abnormal looking tissue.

B. 883-BG: strong evidence of staining around abnormal tissue with possible vascular disturbance and a strong local inflammatory with many macrophages/glia stained positively for $m$ CRP. Here we can see an m CRP-loaded blood vessel secreting or leaking in to the local tissue that has become very strongly $\mathrm{m}$ CRP positive (Figure 1A; x 40 and B x 200)-as compared with a more remote area on the same slide where there is no evidence of $m$ CRP staining (C-x 200). In other areas $m$ CRP is found loaded in to intact vessels without obvious leakage (Figure 1D).

C. 916-OCC: shows an example of large vessels containing $\mathrm{m}$ CRP, near to a site of possible infarction/tissue damage; some of which seems to have been able to enter the local surrounding tissue micro-vessels and neurons in association with inflammation.

D. 850-F: Many small to medium sized vessels stained positive for $\mathrm{m}$ CRP (arrows) and what looks like a regional specific build-up of the protein with the local micro-environment strongly inflammatory and macrophage/glia positively stained (Figure 1E \& 1F; DAB brown).

E. 798-PAR: inflammatory brain region with small vessels and macrophages staining positive for $m$ CRP. Large surrounding areas of negatively stained tissue.

F. 954-OCC: small infarcted area surrounded by m CRP-positively stained macrophages and glia, again with other remote regions remaining negatively stained.
G. 963-Par: a region of tissue next to a micro-infarcted area that has stained strongly positive for $\mathrm{m}$ CRP. Micro vessels and inflammatory cells are positively stained (Figure 1G-x 40 and $\mathrm{H}$ x 200 showing macrophages and vessels positive Dab brown).

\section{Discussion}

This is the first study to perform a focused analysis of specific cases of individuals who died with clinical evidence of neurodegenerative conditions-with a view to identifying and localizing vascular abnormalities associated with $\mathrm{m}$ CRP expression. In this simple IHC study, we show strong evidence that a build-up of $m$ CRP can be found in a majority of 10 individuals presenting with neurodegenerative complications. In addition, this m CRP staining is frequently associated with regions of neuro-inflammation with macrophages and glial cells staining positive as well as small to medium sized blood vessels. Strikingly, we show an image of what appears to be $\mathrm{m}$ CRP un-loading from the site of a larger vessel in to the local parenchyma where it has become associated with existing cells. Monomeric CRP is now known to elicit a pro-inflammatory influence upon macrophages and glia, inducing nitric oxide synthase Sproston NR et al. [15] and TNF- $\alpha /$ IL-1 $\beta$ amongst other cytokines Slevin et al. [15]. Hence within the brain after trauma or in hypoxic conditions, penetration of $\mathrm{m}$ CRP may enhance pro-inflammatory micro-environment causing more damage. Additionally, there is some evidence to support a relationship between CRP/m CRP expression and a direct effect on neurodegeneration and dementia, including beta amyloid plaque formation. In regard to the possible relationship to angiogenesis, aberrant angiogenesis associated with formation of none-patent or leaky micro vessels as part of a regenerative or restorative effort within damaged brain tissue only serves to permeabilize the parenchyma thereby further increasing immune cell infiltration and so on and so forth Badimon et al. [16]. Her we provide solid evidence in support of an important role for $\mathrm{m}$ CRP in creation of and/or perpetuation of inflammation within damaged brain tissue and the vascular compartment, when damaged may provide an access point for leakage of $m$ CRP that may enhance or pre-dispose individuals to the development of dementia or other cognitive deficiencies (Figure 1). 


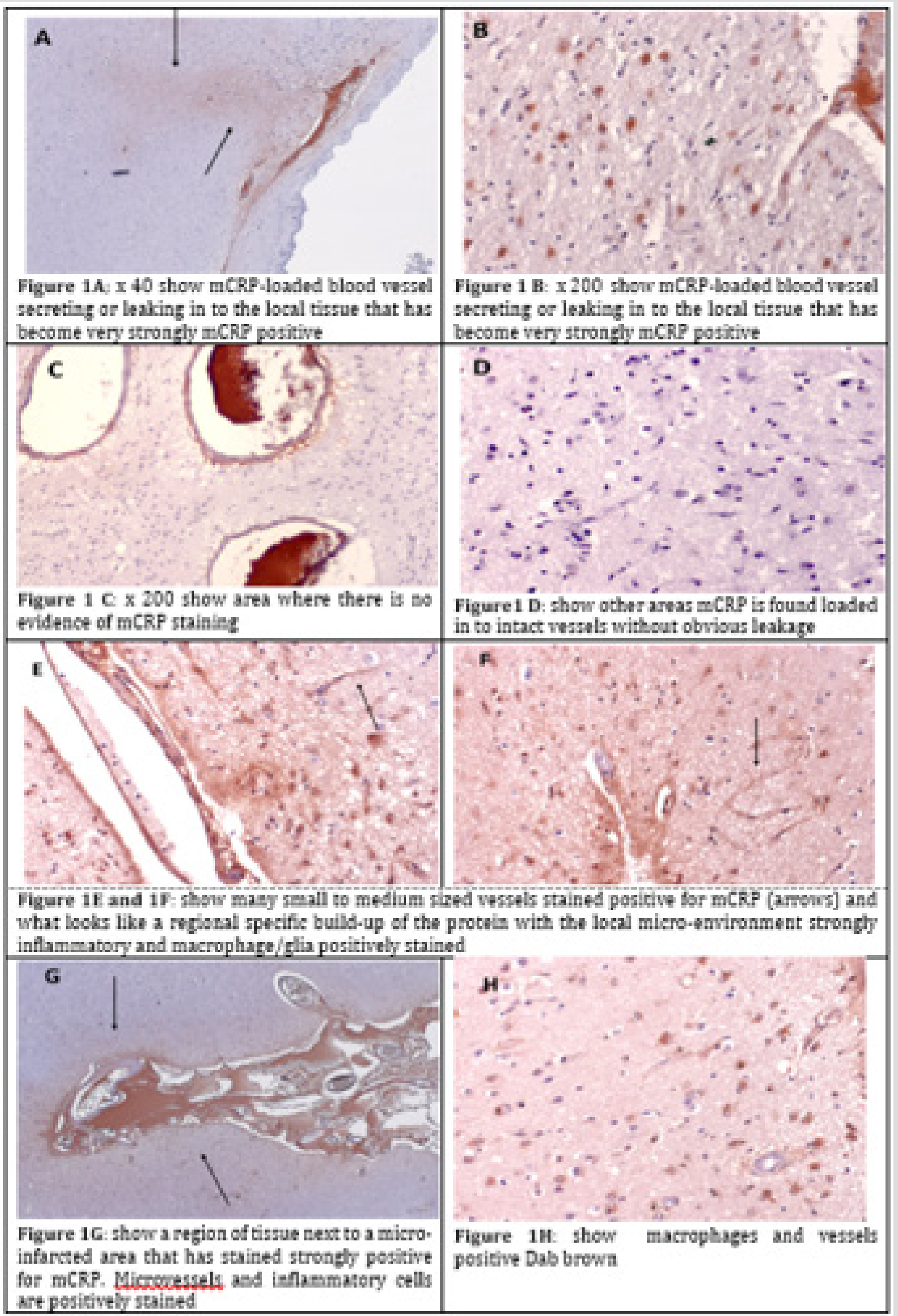

Figure 1: Show the areas $\mathrm{m}$ CRP accumulation. 
A modulator of the proposed target (m CRP) provides a completely novel approach to enable protection from development of Va D (and associated AD) after stroke. This would be the first attempted therapy to target a key molecule associated with vascular damage after stroke and that is strongly linked to neurodegenerative pathology. This is primarily a preventative/ protective strategy rather than a treatment for those already presenting with VaD/AD. This agrees with prior experience in $\mathrm{AD}$ where most of the therapies are started probably too late in the degenerative process. These outcomes high spot the probable of targeted antibodies and macromolecule therapeutics to inhibit the linking of $\mathrm{m}$ CRP by inhibition of interaction of membrane and following stimulation of cellular cascade systems, which yield the pro-inflammatory effects related with monomeric CRP [17]. The Complementarity-determining regions (CDR) of antibody binds with $\mathrm{m}$ CRP, namely; The patent of new antibody has deduced Fab amino acid (AA)-sequences Heavy chains (IgG-gamma): (HVCDR1SMGFH, HVCDR2- FISSRLTIY, HVCDR3-ADTRLT) And Light chains (IgG-gamma): (LVCDR1- KASSVPYMH, LVCDR2- RANDTSNS, LVCDR3- LQDEPRTF) Figure 2.

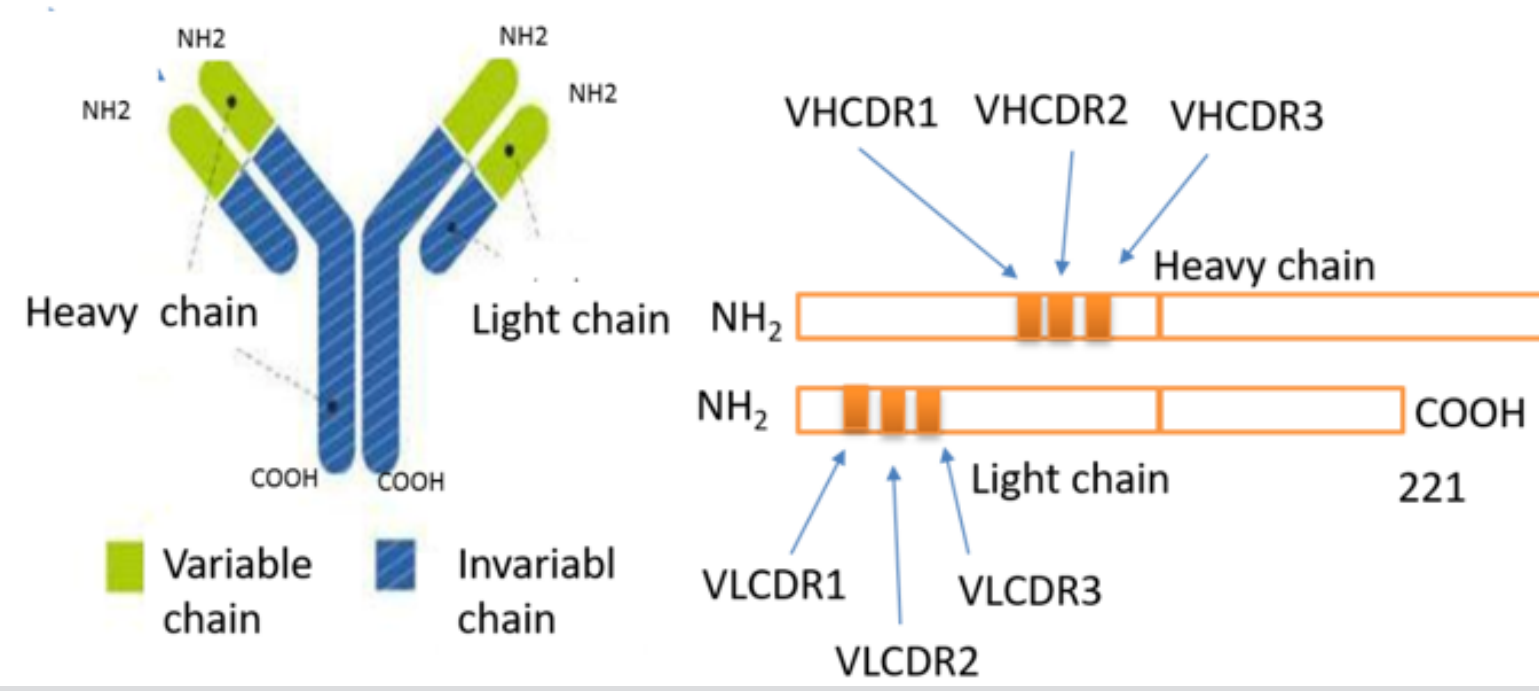

$\mathrm{COOH}$

Figure 2: The Complementarity-determining regions (CDR) of m CRP antibody.

\section{Conclusion}

For the first time, here we have provided evidence of $m$ CRPbuild-up within small and medium sized micro-vessels within the brain tissue localized to stroke. We capture the release into brain parenchymal tissue of $\mathrm{m} \mathrm{CRP}$ and a concomitant activation of regional and focal blood vessels where they are exposed to this protein. This work provides further evidence to support a prominent role for $\mathrm{m}$ CRP in induction of inflammation and neurodegenerative capability after stroke and brain damage. Also, we introduce pharmacological agents with characteristic a preventative, protective and treatment for AD.

\section{Acknowledgement}

This research was reinforced by the Applied Medical Sciences College, Majmaah University, Majmaah 11952, P.O box 1405, Saudi Arabia. We would like to express our gratitude toward RA (r.albaradie@mu.edu.sa) supervisor of the Stroke chair, Majmaah university and Dr. Khalid Bin Saad Al Muqrin, Rector, Majmaah University, and Deanship of Research, Majmaah University for providing the necessary support and assistance for completing this study. This work was carried out under project number 38/130 Majmaah university.

\section{References}

1. HC Hendrie (1998) Epidemiology of dementia and Alzheimer's disease. Am J Geriatr Psychiatry 6(2 Suppl. 1): S3-18.

2. NI wamoto, E Nishiyama, J Ohwada, H Arai (1994) Demonstration of CRP immunoreactivity in brains of Alzheimer's disease: immunohistochemical study using formic acid pretreatment of tissue sections. Neurosci Lett 177(1-2): 23-26.

3. M Slevin, S Matou-Nasri, M Turu, A Luque, N Rovira, et al. (2010) Modified C-reactive protein is expressed by stroke neovessels and is a potent activator of angiogenesis in vitro. Brain Pathol 20(1): 151-65.

4. Montagne A, DA Nation, MD Sweeney, AW Toga, BV Zlokovic, et al. (2016) Brain imaging of neurovascular dysfunction in Alzheimer's disease. Acta Neuropathol 131(5): 687-707.

5. JB Toledo, SE Arnold, K Raible, J Brettschneider, SX Xie, et al. (2013) Contribution of cerebrovascular disease in autopsy confirmed neuro degenerative disease cases in the National Alzheimer's coordinating centre. Brain 136(pt 9): 2697-706.

6. M Slevin, L Donghui, M Al-Hsinawi, R Al-Baradie, J Krupinski (2017) Expression of Monomeric C-Reactive Protein in Infarcted Brain Tissue from Patients with Alzheimer's Disease Turk Patoloji Derg 33: 25-29.

7. Selles, M Clara, Oliveira, M Mauricio, Ferreira, T Sergio (2018) Brain Inflammation Connects Cognitive and Non-Cognitive Symptoms in Alzheimer's Disease. J Alzheimer's Dis 64(S1): 313-327.

8. SP Rensma, TT van Sloten, LJ Launer, CDA Stehouwer (2018) Neuroscience and Biobehavioral Reviews. Neurosci Biobehav Rev 90: 164-173. 
9. H V Vinters, C Zarow, E Borys, JD Whitman, S Tung, et al. (2018) clinicopathologic and genetic considerations. Vascular dementia 44(3): 247-266.

10. YY Luan, YM Yao (2018) The Clinical Significance and Potential Role of C-Reactive Protein in Chronic Inflammatory and Neurodegenerative Diseases. Front Immunol 9: 1302.

11. E Boras, M Slevin, M Yvonne Alexander, A Aljohi, S Matou-Nasri (2014) Monomeric C-reactive protein and Notch-3 co-operatively increase angiogenesis through PI3K signaling pathway Cytokine 69 (2): 165-179.

12. SS Mirra, A Heyman, D McKeel, SM Sumi, BJ Crain, et al. (1991) The Consortium to Establish a Registry for Alzheimer's Disease (CERAD). Part II. Standardization of the neuropathologic assessment of Alzheimer's disease. Neurology 41(4): 479-486.

13. H Braak, I Alafuzoff, T Arzberger, H Kretzschmar, K Del Tredici (2006) Staging of Alzheimer disease-associated neurofibrillary pathology using

ISSN: 2574-1241

DOI: 10.26717/BJSTR.2019.17.003062

AM Algohary. Biomed J Sci \& Tech Res

(C) 1 This work is licensed under Creative

Submission Link: https://biomedres.us/submit-manuscript.php paraffin sections and immunocytochemistry. Acta Neuropathol 112(4): 389-404.

14. TJ Montine (2012) National Institute on Aging; Alzheimer's Association, National Institute on Aging-Alzheimer's Association guidelines for the neuropathologic assessment of Alzheimer's disease: a practical approach. Acta Neuropathol 123(1): 1-11.

15. R Nicola, Sproston, M El Mohtadi, M Slevin, W Gilmore (2018) The Effect of C-Reactive Protein Isoforms on Nitric Oxide Production by U937 Monocytes/Macrophages. Front Immunol 9: 1500.

16. L Badimon, E Peña, G Arderiu, T Padró, M Slevin, et al. (2018) Reactive Protein in Atherothrombosis and Angiogenesis. Front Immunol 9: 430.

17. R Albaradei, AM Alsulaiman, YH Babair, A M Algohary, MK Alfinisan, et al. (2018) Synthesis of antibody of monomeric C-reactive protein for treatment of dementia: King Abdulaziz City for Science and Technology (KACST) KSA patent number 6202. 